\section{Compact C language Fourier analysis on small computers}

\section{PHILLIP L. EMERSON \\ Cleveland State University, Cleveland, Ohio}

The $\mathrm{C}$ language is well suited for the programming of small computers for data collection in real-time laboratory research, so it is becoming convenient to code some of the analysis programs also in $\mathrm{C}$. The pair of $\mathrm{C}$ routines presented here is for simple applications of the fast Fourier transform (FFT). These routines are written compactly for small analyses. They are not optimally efficient, and they are constrained by some limitations that can be transcended by using commercially available FFT programs, such as those reviewed by Eddy and Bremner (1983). Nevertheless, they have the advantage that they are easily incorporated as parts of a $\mathrm{C}$ program whose main purpose is something other than Fourier analysis. Being in source-code form, these routines are easy to adapt for various purposes.

Such adaptations require some acquaintance with the C language. Kernighan and Ritchie's (1988) book can be consulted for the general principles of $\mathrm{C}$ programming, and the software documentation for a particular implementation of $\mathrm{C}$ usually covers specific exceptions and innovations. As an alternative, a main calling program is included here that can be used in many on-line applications without additional programming.

Potential users who have little acquaintance with Fourier analysis may want to consult Kaplan (1983), who explained it in the familiar terms of correlations and variance components. Fourier analysis is fundamentally the same as trend analysis using orthogonal polynomials, except that periodic sine and cosine components are used in place of the polynomial components, such as those that are linear, quadratic, and cubic. Fourier analysis is often used for analyzing cyclic data, such as EEG, speech, animal sounds, and slower biological rhythms. Meaningful analysis requires experimental design planning that is peculiar to Fourier analysis and, often, some preprocessing of the data before the FFT is performed (Blackman \& Tukey, 1958; Glass, Wilson, \& Gottman, 1975).

The Algorithm and Program Elements. In the standard terminology for variations on the fundamental FFT algorithm (Liu, 1975), the method used here is frequency decimation with postshuffling. It was coded first in BASIC (Emerson, 1980) and now in C, directly from the signal flow graph of Figure 10 in Cochran et al. (1967). The radix is 2 , for the simplest computational algorithm, and the computations are done in place in the sense that the transform ends up in the same memory arrays in which

The author's mailing address is Department of Psychology, Cleveland State University, Cleveland, $\mathrm{OH} \mathbf{4 4 1 1 5 .}$ the original data were stored. An $A$ array is used for the real components and $B$ for the imaginary. Actually, both arrays hold real quantities, but those in the $B$ array are assumed implicitly to have the coefficient $\sqrt{-1}$ so that each $(A, B)$ pair represents a complex number. 'The in$\operatorname{dex}, i$, runs from 0 to $n-1$, where $n$ is the number of elements in each of the two arrays. Because the radix is $2, n$ must be an integral power of 2 . The main computations of the FFT are performed by the freqdec() routine shown in Listing 1 . Before calling freqdec( ) for the first time with a new value of $\boldsymbol{n}$, the calling program must create the $q[]$ array, which is a quarter-wave table of cosines to be used in the FFT calculations. The $i$ th element of $q[]$ is $\cos (2 \pi i / n)$, for $0 \leq i \leq n / 4$. The $q$ array is not modified by calls to freqdec( ). The direction of the transform, forward (time to frequency) or inverse (frequency to time), is controlled by the argument sgn in the call to freqdec() (sgn $=1$ for forward ; sgn $=-1$ for inverse).

Assume that a time series is to be transformed, and that it is obtained as a sequence of equally spaced measurements on some quantitative variable. As soon as $n$ is known, the $q$ array can be constructed. Then the elements of the time series should be inserted in their natural order into the $A$ array, starting at $A_{0}$. If possible, the length of the time series should be made an integral power of 2 in the experimental design. If that cannot be done, then the series will not fill the $A$ array, and a data window of the length of the actual time series should be applied, with the trailing empty $A$ elements set equal to zero. This data window should be tapered toward zero on both ends, as is the Hamming window, which will be used in an example. $^{2}$ All of the $B$ elements should be set equal to zero. The freqdec() routine should then be called, and postshuffling should be done using bitrev(), as will be illustrated. Note that the $A$ and the $B$ arrays will contain the complex exponential form of the FFT. For some purposes, the coefficients $\left(a_{j}, b_{j}\right)$ in the trigonometric form of the Fourier series are needed. This series is

$$
a_{0}+\sum\left[a_{j} \cos (2 \pi i j / n)+b_{j} \sin (2 \pi i j / n)\right],
$$

where the summation starts at $j=1$. These coefficients are obtainable from the elements of the $A$ and $B$ arrays, after postshuffling, as

$$
a_{0}=A_{0} / n, \quad b_{0}=0,
$$

and

$$
a_{j}=\left(A_{j}+A_{n-j}\right) / n, \quad b_{j}=\left(-B_{j}+B_{n-j}\right) / n,
$$

for $0<j<n / 2$. The power (energy, or variance component) at frequency $j /(n \Delta t)$, where $\Delta t$ is the spacing between time points, can then be computed as $a_{j}^{2}+b_{j}^{2}$, and the amplitude is the square root of the power.

A Calling Program. The main program of Listing 2 can be used for test purposes and for some real applications. It compiles with popular $\mathrm{C}$ implementations, such 
LISTING 1

The FFT Subroutines

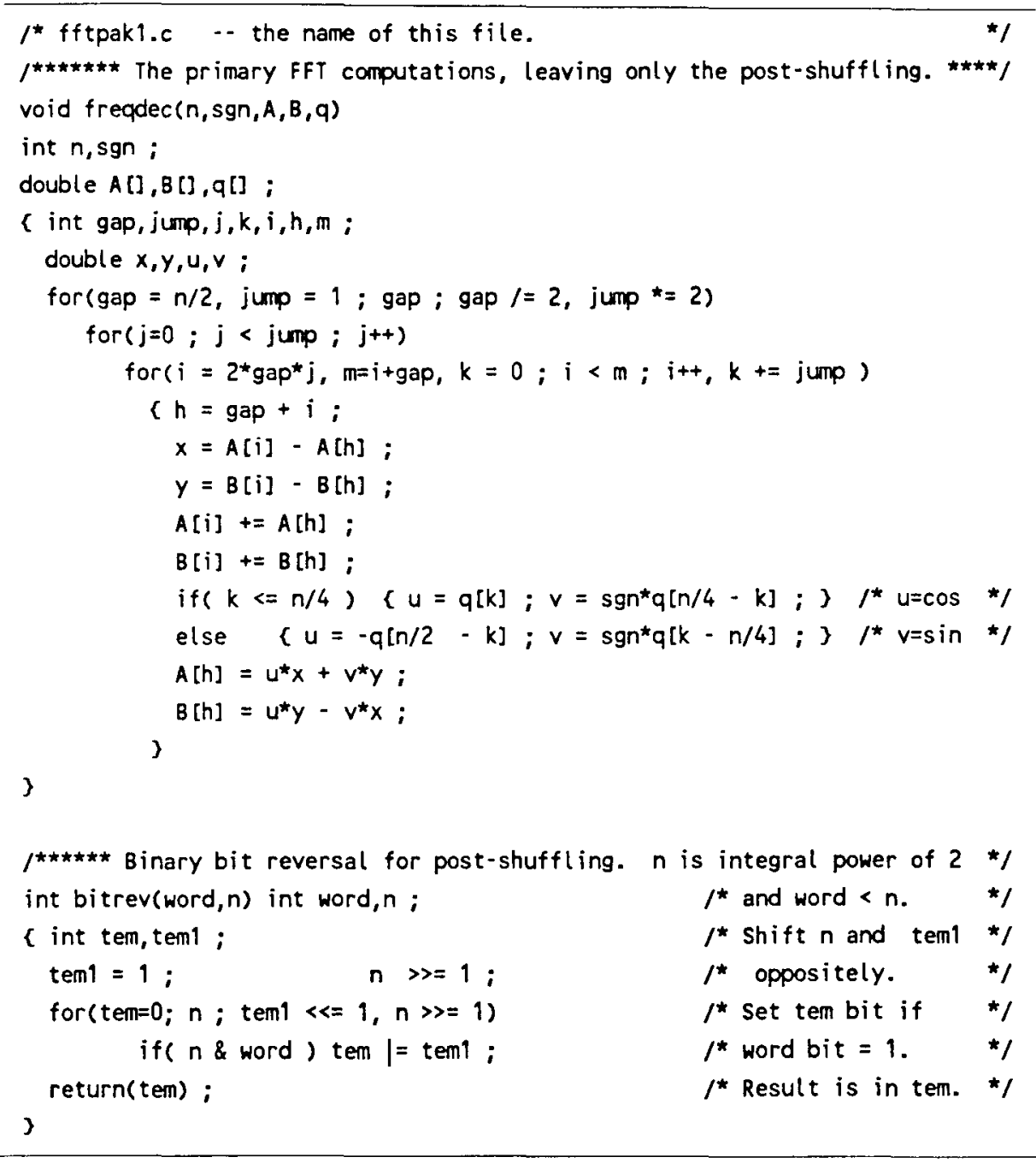

LISTING 2

A Main Program Calling the FrT Routines with Input Data from Keyboard or File, and Output to Screen or File

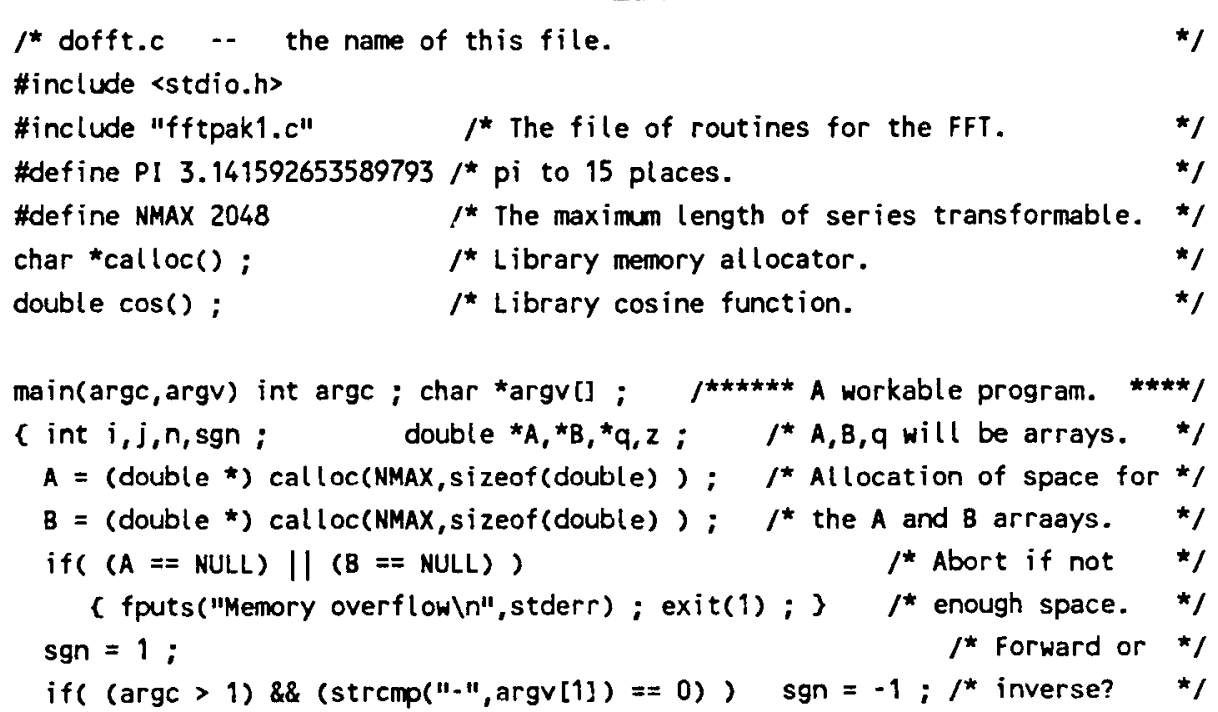


LISTING 2 (Continued)

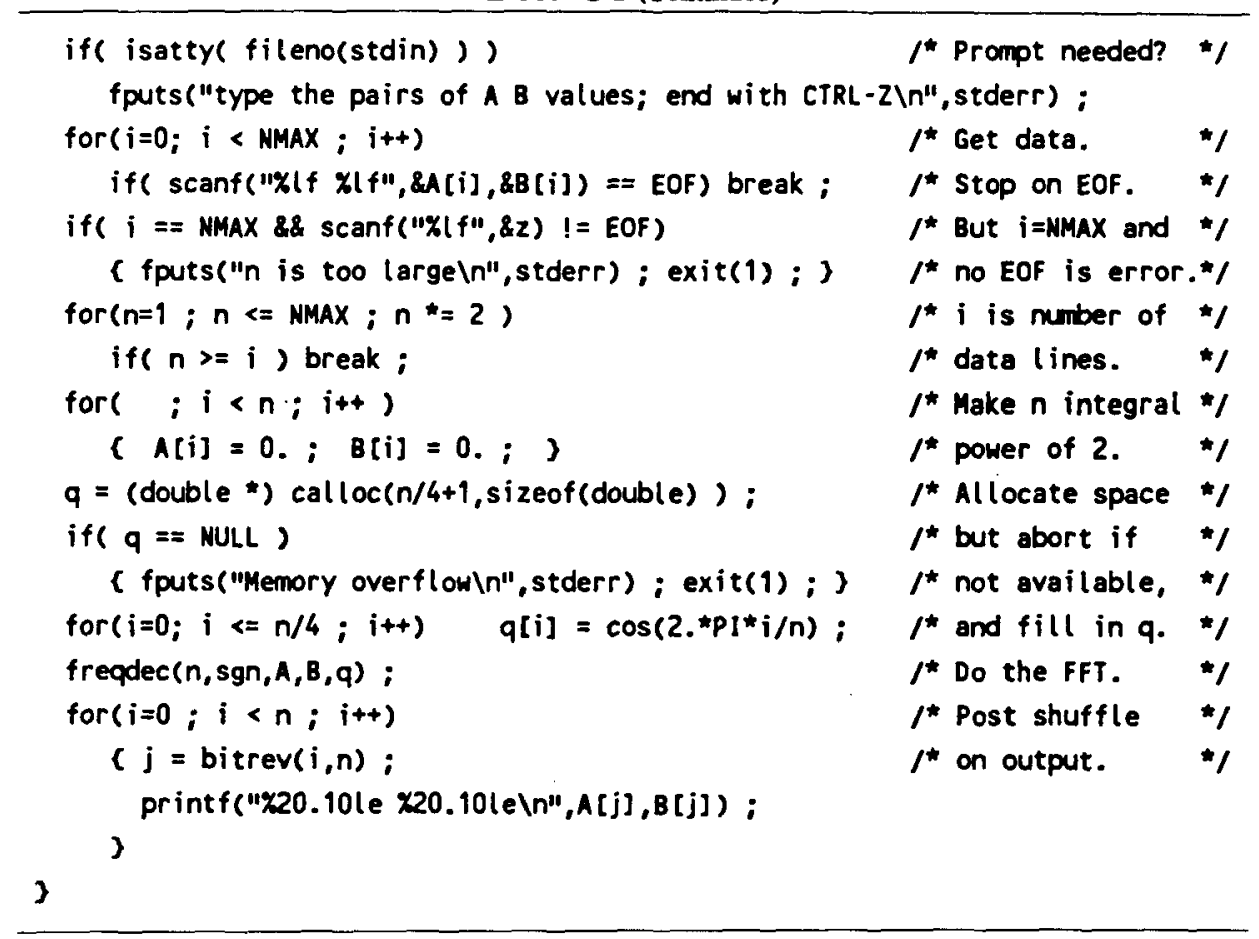

as Microsoft C, Datalight C, and Borland Turbo C. It performs a single FFT and then exits to the operating system. It is a minimal FFT program, but it is designed to be used with readily available supporting facilities that make it quite flexible. The input/output redirection and piping capabilities of MS-DOS are very helpful, and the data-manipulation programs in Perlman and Horan's (1986) UNIX|STAT package greatly extend the flexibility.

After the program of Listing 2 is compiled and linked, the result will be the executable file, DOFFT.EXE. There is only one command line option. If the program is invoked simply by typing DOFFT after the MS-DOS prompt, a forward FFT will be performed. If it is invoked with an appended space and minus sign (DOFFT -), an inverse transform will be performed. After the DOFFT command is issued to MS-DOS, the data are typed on the keyboard, and the results appear on the screen. On input as well as output, the data are in only two columns, which are the contents of the $A$ and $B$ arrays.

For realistic applications in which data sets are larger, an input file, say INFILE, can be created using a text editor, or produced directly by some data-collection program. The contents should be, as before, a pair of columns for the $A$ and $B$ arrays. Care should be taken to include no blank lines or extraneous comments in this file (even at the end) since lines are counted to determine the number of elements in the series to be transformed. The command to transform the data in this file would be DOFFT $<$ INFILE, and the output would come to the screen. To redirect the output to a disk file, say OUTFILE, the command would be DOFFT < INFILE > OUTFILE.

Consider two examples in which Perlman and Horan's (1986) UNIX|'STAT manipulation programs are quite useful. In the first, the initial time series is an 8-point square wave that will be transformed and then detransformed. The series can be entered into INFILE with the $A$ column containing four 1 values followed by four 0 values, and the $B$ column containing all 0 values. The command to do the forward transform is DOFFT < INFILE > OUTFILE. Before the inverse transform is applied to OUTFILE, all elements of $A$ and $B$ need to be divided by $n$ ( 8 in this case). The UNIX|STAT command, dm $\times 1 / 8 \times 2 / 8<$ OUTFILE > OUT1, will accomplish this, putting the results in the new file, OUT1. The inverse transform then can be done with the command DOFFT $-<$ OUT1, from which the output comes to the screen. Except for rounding errors, the resulting numbers should be the same as those contained in the original INFILE. These operations are those needed to transform a series, and then detransform it.

As the second example, assume the existence of a file, DATA, that contains a time series of 200 real quantitative measurements in a single column. Note that 200 is not an integral power of 2 and that the program of Listing 2 will expand the length to 256 elements, appending zeros in the last 56. Assume, then, that this series is to be preprocessed before transforming, with a Hamming time window (Blackman \& Tukey, 1958) of length $200 .^{3}$ Thus, it is reasonable to create a derived file that will con- 
tain the required second column of zeros for the $B$ array, as well as the preprocessed data in the $A$ array. A UNIX|STAT command to accomplish this is

$$
\begin{array}{ll}
\operatorname{dm} & x 1 *(.54-.46 * \cos (2 * 3.14159 *(\text { INLINE }-1) / 200)) \\
& 0<\text { DATA }>\text { HDATA }
\end{array}
$$

The new file, HDATA, is ready for input to DOFFT by a command such as DOFFT < HDATA > RESULTS. The FFT will appear in the newly created file, RESULTS. This example can be extended to illustrate the computation of the power spectrum from the FFT in the RESULTS file. Most of the calculations can be done by the UNIX|STAT command

$$
\begin{aligned}
\mathrm{dm} & (\mathrm{x} 1 / 128) *(\mathrm{x} 1 / 128)+(\mathrm{x} 2 / 128) *(\mathrm{x} 2 / 128) \\
& <\text { RESULTS > POWER }
\end{aligned}
$$

which places the output in a file called POWER. These output values are correct for all $n / 2$ of the $a_{j}^{2}+b_{j}^{2}$ elements, except that the first element must be divided by 4 to give the correct value of the dc power.

Limitations. The radix of 2 makes the programming of the algorithm of Listing 1 simple, but it also leads to the requirement that the series to be transformed have a length that is an integral power of 2 .

The value of NMAX in Listing 2 is 2,048, which is the maximum length of series that can be transformed. The maximum can be doubled if the $A$ and $B$ arrays are changed from type double to type float. This maximum is designed to be safe with most $\mathrm{C}$ compilers implemented with MS-DOS and with machines having at least $192 \mathrm{~K}$ of memory. For larger memories and some compilers, it would be possible to extend this maximum substantially, but the disadvantage is that some modifications probably would be needed to accommodate "far pointers," which are not implemented in the same ways by different implementations of MS-DOS C.

The accuracy of the computations is about 14 significant figures when short sequences are transformed. For longer sequences, the greater numbers of calculations lead to greater accumulations of round-off errors. A notable source of inaccuracy for multiple-file operations with the main program of Listing 2 is the formatted output. The specification of \%20.10le provides for about 11 significant digits, whereas about 15 are available with the standard double data type. Increasing the format length, however, slows down the input and output.

\section{REFERENCES}

Blackman, R. B., \& TUKey, T. W. (1958). The measurement of power spectra. New York: Dover.
Cochran, W. T., Cooley, J. W., Favin, D. L., Helms, H. D., Kaenel, R. A., Lang, W. W., Maling, G. C., Jr., Nelson, D. E., RADER, C. M., WELCH, P. D. (1967). What is the fast Fourier transform? IEEE Transactions on Audio Electroacoustics AU-15(2), 45-55. (Reprinted in Liu, 1975.)

EDDY, D. R., BREMNER, F. J. (1983). Computer packages that contain the FFT. Behavior Research Methods \& Instrumentation, 15, 254-257.

Emerson, P. L. (1980, July). Fast Fourier transform fundamentals and applications. Creative Computing, 6, 58-63.

Glass, G. V., Wilson, V. L., Gottman, J. M. (1975). Design and analysis of time-series experiments. Boulder: Colorado Associated University Press.

KAPLAN, H. L. (1983). Correlations, contrasts, and components: Fourier analysis in a more familiar terminology. Behavior Research Methods \& Instrumentation, 15, 228-241.

Kernighan, B. W., \& Ritchie, D. M. (1988). The C programming language (2nd ed.). Englewood Cliffs, NJ: Prentice-Hall.

LiU, B. (Ed.). (1975). Digital filters and the fast Fourier transform. New York: Halstead.

Perlman, G., \& Horan, F. L. (1986). Report on UNIX'STAT release 5.1 data analysis programs for UNIX and MSDOS. Behavior Research Methods, Instruments, \& Computers, 18, 168-176.

\section{NOTES}

1. For most of the simple applications that might be envisioned, the time series would consist of purely real numbers, and the transform would therefore be perfectly symmetrical. Thus, more memory space is used than would be required in principle. A reviewer suggested that the extra memory space might be usable in special batch operations by transforming two independent real series in the same operation, starting with one in the $A$ array and the other in the $B$ array. Retrieval of the separate results would require the separation of even-symmetric from oddsymmetric components, and some other minor adjustments. A related possibility is the generalized spectral analysis of a time series of twoelement vectors, such as the plane coordinates of a moving object.

2. End tapering seems like the best general-purpose way to treat the situation in which the length of the observed time series is not an integral power of 2 , although other treatments might be better in some special applications.

3. The Hamming window is symmetrical and smooth with maximal transmission at its center, tapering nearly to zero on each end. Some other function with these characteristics might serve as well. A tapered data window often is used even when the length of the time series is an integral power of 2 , to temper the possible discontinuity between the end and beginning, which are adjacent in the periodic representation assumed in using the FFT. Although they are not adjacent in this example, the tapered ends temper the possible discontinuities at the boundaries of the separating gap. A sharp discontinuity of the dc level of the time series creates a splattering of power over many frequencies far up and down the spectrum. If the time series contains true discontinuities, the splattering is correct, in a sense, although it makes the Fourier analysis difficult to interpret. If the discontinuities are merely artifacts of end mismatch, on the other hand, end tapering works well to combat the splatter and reveal true periodic components. 